\title{
MiR-30c Plays Diagnostic and Prognostic Roles and Mediates Epithelial-mesenchymal Transition (EMT) and Proliferation of Glioma by Affecting Notch1
}

\section{Mengkao Li}

Taian Central Hospital

Wenzhi Liu

Taian Central Hospital

Jin Xu ( $\nabla$ doctorxujin66@163.com)

Shandong Cancer Hospital and Institute, Shandong First Medical University and Shandong Academy of Medical Sciences

\section{Research Article}

Keywords: miR-30cmiR-30c, epithelial-mesenchymal transition (EMT) , glioma, Notch1

Posted Date: August 20th, 2021

DOI: https://doi.org/10.21203/rs.3.rs-799131/v1

License: (c) (i) This work is licensed under a Creative Commons Attribution 4.0 International License. Read Full License 


\section{Abstract}

miR-30c functions as a tumor suppressor gene in the majority of tumors, including glioma. In our study, we discovered that the expression levels of miR-30c in glioma tissues and plasma prior to operation were lower than that in normal brain tissue following brain injury decompression and plasma in healthy volunteers. The low expression of miR-30c was closely aligned with WHO grade, tumor size, PFS, and OS. Additionally, the miR-30c expression level of tumor tissue was positively correlated with the levels found in preoperative plasma. In cell biology experiments, miR-30c was discovered to inhibit EMT, proliferation, migration, and the invasion of glioma cells. The database of miRNAs target genes, real-time quantitative PCR, western blot, and dual luciferase reporter assay demonstrated that Notch1 is the direct target gene of miR-30c. The inhibitor and shRNA-Notch1 were cotransfected into glioma cells, which illustrated that shRNA-Notch1 could reduce the enhancement of inhibitors in EMT, proliferation, migration, and the invasion of glioma cells. Therefore, we believe that when utilized as a tumor suppressor gene, miR-23a can inhibit EMT, proliferation, migration, and invasion of glioma cells by directly acting on Notch1 at the post-transcriptional level, and it is a potential diagnostic and prognostic marker.

\section{Background}

Gliomas are among the most common malignant tumors present in the central nervous system that arise from glial cells or precursor cells. According to World Health Organization (WHO) classifications, gliomas are categorized into four grades. Grade 1 and grade 2 gliomas are considered low grade, and grade 3 and grade 4 gliomas are high grade. Grade 4 gliomas are labeled glioblastomas (GBM). Despite active and effective treatment, the median survival time of GBM patients is still less than 20 months ${ }^{1}$. Therefore, the treatment of glioma poses a great challenge. In order to establish effective treatment methods, it is paramount to clarify the molecular mechanisms present in the occurrence and development of glioma.

Epithelial mesenchymal transition (EMT) is defined as the biological process through which epithelial cells become cells with a mesenchymal phenotype ${ }^{2}$. In the EMT process, epithelial cells lose cell polarity, decrease contact with surrounding cells and stromal cells, decrease interaction between cells, enhance cell migration and mobility, alter the cell phenotype, and lose the epithelial phenotype. Regarding the latter, one such example is a decrease in E-cadherin levels, which leads to decreased cell adhesion ${ }^{3}$ and causes cells, as such, to obtain the characteristics of invasion and metastasis ${ }^{4}$. Simultaneously, the number of cells which obtained mesenchymal phenotypes, those including Vimentin and N-cadherin, were raised ${ }^{5,6}$.

The Notch signaling pathway is a common signaling pathway involved in EMT process control 7,8 . The Notch signaling pathway is comprised of the Notch receptor, Notch ligand, CSL-DNA binding protein, and Notch regulatory molecules. A signal transmitted between adjacent cells through the Notch receptor can regulate cell differentiation, proliferation, and apoptosis. The Notch receptor is a single transmembrane protein encoded by the Notch gene. Four kinds of Notch receptors (Notch 1, 2, 3, and 4) have been located in mammals, including humans. In the process of malignant tumorigenesis, the Notch signaling pathway 
plays the carcinogenic roles, leading to cell proliferation ${ }^{9}$, differentiation ${ }^{10}$, apoptosis ${ }^{11}$, and process imbalance, all of which contribute to the occurrence and development of malignant tumors. In glioma, Notch1 was highly expressed ${ }^{12}$. In glioma cells, Notch1 expression is up-regulated, and plays a role in tumor progression. Notch1 may also function as an independent prognostic marker that is both separate from and additional to the WHO classifications ${ }^{13}$. Notch1 also promotes EMT in a variety of tumors, including glioma $14,15,16$.

miRNAs are 19-22 nucleotides noncoding RNAs, which act as an oncogene or tumor suppressor gene by connecting the 3 '- UTR region of the target gene and regulating the expression of related proteins at the post-transcriptional level. miRNAs function in regulating cell proliferation ${ }^{17}$, apoptosis ${ }^{18}$, invasion ${ }^{19}$, angiogenesis ${ }^{20}$, and the cell cycle ${ }^{21}$, and in turn participate in the occurrence and progress of an assortment of tumors. miR-30c plays different roles in different tumors $22,23,24$, but there is no comparable research into glioma. In this study, the authors explore the mechanism of miR-30c in glioma, and offer a theoretical basis for the diagnosis and treatment of glioma.

\section{Results}

\section{The expression of miR-30c was decreased in tissues and plasma from patients with glioma.}

The expression of miR-30c was determined using qRT-PCR. As detailed in Fig. 1, the relative expression of miR-30c in 66 cases of glioma tissues was $3.077 \pm 0.282$, while the relative expression of miR-30c in 9 patients with traumatic brain injury was $9.352 \pm 0.190$. The expression levels of miR-30c in glioma tissues were less than that of normal brain injury tissue, and the difference was statistically significant $(P<0.01)$ (Fig. 1A). As detailed in Table 1, the expression level of miR-30c in glioma tissues was closely-correlated with WHO grade and tumor size $(P<0.01)$, but not with the gender, age, extent of resection, and KPS scores $(P>0.05)$ of patients.

Table 1

The connection between miR-30c levels in the tissue and plasma, and the clinicopathologic characteristics of glioma patients (* Indicates $\mathrm{P}<0.05$, ** Indicates $\mathrm{P}<0.01$ ). 


\begin{tabular}{|c|c|c|c|c|c|}
\hline Characteristics & number & Tissue & $P$ value & Plasma & $P$ value \\
\hline \multicolumn{6}{|l|}{ Gender } \\
\hline Male & 41 & $3.132 \pm 0.287$ & 0.806 & $0.804 \pm 0.068$ & 0.865 \\
\hline Female & 25 & $2.987 \pm 0.586$ & & $0.827 \pm 0.135$ & \\
\hline \multicolumn{6}{|l|}{ Age } \\
\hline$<50$ & 32 & $3.042 \pm 0.330$ & 0.906 & $0.869 \pm 0.099$ & 0.406 \\
\hline$\geq 50$ & 34 & $3.110 \pm 0.456$ & & $0.759 \pm 0.087$ & \\
\hline \multicolumn{6}{|l|}{ Tumor size } \\
\hline$<5 \mathrm{~cm}$ & 27 & $4.133 \pm 0.444$ & $0.001^{\star *}$ & $1.073 \pm 0.100$ & $0.001^{* *}$ \\
\hline$\geq 5 \mathrm{~cm}$ & 39 & $2.346 \pm 0.320$ & & $0.633 \pm 0.075$ & \\
\hline \multicolumn{6}{|l|}{ WHO grade } \\
\hline I & 3 & $5.353 \pm 0.214$ & 0.001 ** & $1.423 \pm 0.054$ & $0.003^{* *}$ \\
\hline ॥ & 17 & $4.215 \pm 0.403$ & & $1.034 \pm 0.075$ & \\
\hline III & 16 & $3.503 \pm 0.652$ & & $0.895 \pm 0.119$ & \\
\hline IV & 30 & $1.977 \pm 0.368$ & & $0.583 \pm 0.106$ & \\
\hline \multicolumn{6}{|c|}{ Extent of resection } \\
\hline$<95 \%$ & 17 & $3.046 \pm 0.862$ & 0.949 & $0.720 \pm 0.174$ & 0.409 \\
\hline$\geq 95 \%$ & 49 & $3.088 \pm 0.243$ & & $0.845 \pm 0.065$ & \\
\hline \multicolumn{6}{|l|}{ KPS score } \\
\hline$<80$ & 32 & $3.073 \pm 0.502$ & 0.989 & $0.706 \pm 0.074$ & 0.114 \\
\hline$\geq 80$ & 34 & $3.081 \pm 0.285$ & & $0.914 \pm 0.105$ & \\
\hline
\end{tabular}

We also identified the expression levels of miR-30c in the pre-surgery plasma of patients with glioma and healthy volunteers. The expression level of miR-30c in glioma patients was $0.813 \pm 0.066$, and in healthy volunteers it was $2.403 \pm 0.136$. The difference was statistically significant $(P<0.01)$ (Fig. 1B). As illustrated in Table 1, the expression level of miR-30c in plasma of glioma patients was closely related to WHO grade and tumor size $(P<0.01)$, but not to gender, age, extent of response, and KPS score $(P>$ 0.05). The expression levels of miR-30c in tumor tissue were positively correlated with levels found in plasma by the Pearson correlation test $(r=0.868, P=0.002)$ (Fig. $1 C)$. The expression levels of miR-30c in tissues and plasma were consistent.

In order to discern if miR-30c can indicate the prognosis of glioma in patients, we determined the expression levels of miR-30c in paraffin-embedded glioma tissues, and we analyzed the correlation 
between the expression level of miR-30c, progress free survival (PFS), and overall survival (OS) using the Kaplan-Meier curve. Fig. 1D, E showed that PFS and OS of patients with low expressions of miR-30c were significantly shorter than those with a higher expression of miR-30c. Thus, it can be inferred that miR-30c could predict the prognosis of patients with glioma.

\section{miR-30c inhibits EMT, proliferation, migration, and invasion of glioma cells.}

We then transfected mimics or NC, inhibitor or inhibitor NC, into A172 and U251 glioma cells, respectively. The expression level of miR-30c in the mimics group was significantly more than that in the NC group ( $P$ $<0.01$ ). The expression level of miR-30c in the inhibitor group was significantly less than those from the inhibitor NC group $(P<0.01)$ (Fig. 2A, B).

In the Western blot experiment, the protein quantitation of $\mathrm{N}$-cadherin and Vimentin in the mimics group was lower than those from the NC group $(P<0.01)$, and the protein expressions of E-cadherin in the mimics group was higher than those from the NC group $(P<0.01)$. Regarding the inhibitor group, the protein quantity of $\mathrm{N}$-cadherin and Vimentin was higher than that of the inhibitor $\mathrm{NC}$ group $(\mathrm{P}<0.01)$, and the protein expression of E-cadherin was lower than those in the inhibitor NC group $(P<0.01)$ (Fig. 2C, D). $\mathrm{E}$-cadherin, $\mathrm{N}$-cadherin, and Vimentin are marker genes found in the process of EMT. Therefore, we are confident believe that miR-30c can inhibit the EMT process of glioma cells.

In the cell proliferation experiment, as shown in Fig. $3 \mathrm{~A}, \mathrm{~B}$, the proliferation ability of glioma cells in the mimics group was less than those found in the NC group $(P<0.05, T=48 h ; P<0.01, T=72 h)$. The cell proliferation ability of the inhibitor group was more than the inhibitor NC group $(P<0.05, T=48 h$; $P<$ $0.01, T=72 \mathrm{~h}$ ). Therefore, we have determined that miR-30c can potentially inhibit the proliferation ability of glioma cells.

As seen in Fig. 3C-F, the migration and invasion abilities of A172 and U251 transfected with mimics were significantly less than those in the NC group $(P<0.01)$, whereas the migration and invasion abilities of A172 and U251 cells transfected with the inhibitor were significantly more than those in the inhibitor NC group $(P<0.01)$. Our results suggested that $\mathrm{miR}-30 \mathrm{c}$ has the potential to inhibit the migration and invasion of glioma cells.

\section{Notch1 is a direct target gene of miR-30c in glioma.}

According to TargetScan, miRWalk, and miRanda databases, we predicted that Notch1 might be the direct target gene of miR-30c, and its action site is shown in Fig. 4A. When mimics were transfected into A172 and U251, the expression levels of the Notch1 protein in the mimics group were significantly less than that in the NC group $(P<0.0)$ (Fig. 4B, C). However, there was no significant change in Notch1 mRNA (Fig. 4D). When the inhibitor was transfected into glioma cells, the expression levels of the Notch1 protein 
in the inhibitor group were significantly more than that found in the inhibitor NC group $(P<0.01)$ (Fig. 4B, C). There was no significant change in Notch1 mRNA (Fig. 4D). In the dual luciferase experiment, as shown in Fig. 4E, F, miR-30c could reduce the luciferase activity found in Notch1-WT in A172 and U251 cells, but it had no effect on Notch1-Mut. Our findings suggest that Notch1 is a direct target gene of miR$30 \mathrm{c}$ in glioma.

\section{Notch1 can promote EMT, proliferation, migration, and invasion of glioma.}

shRNA can hinder the expression of Notch1 in glioma cells. In Fig. 5A, qRT-PCR detection revealed that the expression level of Notch1 mRNA following shRNA-Notch1 transfection was notably less than that located in the NC group $(P<0.01)$, which provided a guarantee for the next experiments. In Western blot, as shown in Fig. 5B, C, we can see the protein levels of N-cadherin and Vimentin in the shRNA-Notch1 group were less than that of the NC group $(P<0.01)$, and that the protein levels of E-cadherin in the shRNA-Notch1 group were more than that of the NC group $(P<0.01)$. Fig. $6 A, B$ show that inhibition of Notch1 expression can reduce the proliferation of $A 172$ and $U 251$ cells $(P<0.01)$, and also reduce the expression of Notch1 in glioma cells, which can significantly curtail the cell migration and invasion ability $(P<0.01)$ (Fig. 6C, D). Therefore, Notch1 can promote EMT, proliferation, migration, and invasion of glioma.

\section{miR-30c inhibited EMT, proliferation, migration, and invasion of glioma by reducing Notch1 expression.}

In order to more fully discern the mechanism of miR-30c in glioma, and likewise to clarify that miR-30c can inhibit the EMT process of glioma by reducing the expression levels of Notch1, we implemented the co-transfection experiment. By co-transfecting the miR-30c inhibitor and shRNA-Notch1 into A172 and U251 cells, we uncovered that the miR-30c inhibitor could enhance the proliferation of glioma cells, while shRNA-Notch 1 could attenuate this change $(P<0.01)$ (Fig. 7A, B). Moreover, we discovered that shRNANotch1 can attenuate the effect of miR-30c inhibitor on the migration and invasion of A172 and U251 glioma cells (Fig. 7C-F). The expression of the key factors N-cadherin and Vimentin increased, and the expression of E-cadherin decreased in the EMT process induced by the inhibitor. However, co-transfection of shRNA-Notch1 attenuated the changes of key gene proteins in EMT (Fig. 8A, B). Our results suggest that miR-30c could inhibit EMT, proliferation, migration, and the invasion of glioma cells by directly acting on Notch1.

\section{Discussion}

miRNAs target genes are large in number and wide in variety, and are involved in multiple cellular signal transduction pathways. Not only do they create a complex signal regulatory network, but they also serve 
an assortment of biological purposes in life activities $25,26,27$. In recent years, research has indicated that miR-30c is actively involved in the regulation of malignant tumor signaling pathways, and further, that it acts as a tumor suppressor gene in the majority of malignant tumors, those such as renal cell carcinoma, colorectal cancer, gastric cancer, and breast cancer $28,29,30,31$. In glioblastoma, some studies suggest that miR-30c can hinder the proliferation and migration of tumor cells by affecting Sox $9^{32}$. Our study explored all levels of glioma and discovered that even in low-grade glioma, the expression levels of miR$30 \mathrm{c}$ still decreased to a significant extent, and that those with a higher grade had lower expression levels of miR-30c. The expression level of miR-30c in glioma tissues and preoperative plasma were closely related to WHO grade and tumor size, and the expression level of miR-30c in tissues and plasma remained consistent. Moreover, in paraffin sections, with lower miR-30c, we noted less PFS and OS, and such information can predict the prognosis of glioma patients.

miR-30c is closely related to a variety of cell biological functions. Unlimited cell proliferation is an important biological feature of malignant tumor cells. In this experiment, miR-30c was discovered to hinder the proliferation of glioma cells. EMT is defined as a process by which epithelial tumor cells lose their adhesive ability and obtain the ability of mesenchymal cell migration to promote metastasis and drug resistance. Our research found that increasing the expression level of miR-30c can also reduce the protein expression of $\mathrm{N}$-cadherin and Vimentin, just as it can increase the protein expression of $\mathrm{E}$ cadherin, thereby inhibiting the EMT processes of glioma cells. Additionally, tumor metastasis is a multistage and multi-step orderly process, and the ability of tumor migration and invasion is the premise of tumor metastasis. We deteremined that miR-30c can inhibit the migration and invasion of glioma cells.

Forecasting the database, we predicted that Notch1, which functions as an important element in glioma, may be the direct target gene of miR-30c. By increasing the expression level of miR-30c, the expression of Notch1 protein decreased, while the expression levels of miR-30c was inhibited, the expression of Notch-1 protein increased. But there was no effect on Notch1 mRNA. In the dual luciferase assay, miR-30c reduced the levels of luciferase activity of Notch1-WT in glioma cells but had no effect on Notch1-Mut. Therefore, we believe that Notch1 is the direct target gene of miR-30c and functions post-transcriptionally.

Notch1 plays an instrumental part in glioma cells, with the functions of enhancing stemness and tumorigenicity ${ }^{33}, 34$, promoting tumor growth ${ }^{35}, \mathrm{EMT}^{36}$, and increasing chemotherapy drug resistance ${ }^{37}$. In this study, we used shRNA to inhibit the expression levels of Notch1 in glioma cells, and we revealed that EMT ( $\mathrm{N}$-cadherin and Vimentin decreased, E-cadherin increased), proliferation, migration, and invasion ability of cancer cells were significantly decreased. This further confirmed the carcinogenic effect of Notch1. Afterwards, we co-transfected the miR-30c inhibitor and shRNA-Notch1 into two glioma cell lines. shRNA-Notch1 could attenuate the enhancement effect of inhibitor on EMT, proliferation, migration, and invasion of glioma cells, thus confirming that miR-30c regulates these processes of glioma cells post-transcriptionally by targeting Notch1.

In conclusion, the expression of miR-30c in glioma tissue and preoperative plasma was decreased, and the low expression level was closely tied to WHO grade, tumor size, and prognosis. miR-30c can inhibit 
EMT, proliferation, migration, and the invasion of glioma cells. miR-30c plays the role of tumor suppressor gene by targeting Notch 1 at the post-transcriptional level. Therefore, miR-30c may act as a tumor marker for tumor diagnosis, treatment, and prognosis.

\section{Materials And Methods \\ Clinical Specimens.}

Sixty-six patients with brain glioma who were first diagnosed in Taian Central Hospital (Taian, Shandong, China) from June 2018 to May 2020 were selected, and $2 \mathrm{ml}$ of peripheral venous plasma was collected from each patient prior to surgery. The other 9 normal, non-glioma brain tissue samples were obtained from patients after decompression surgery to treat traumatic brain injury, which was confirmed as normal brain tissue by postoperative pathology. The control plasma samples were collected from 9 healthy volunteers.

Seventy-two cases of brain glioma paraffin specimens were selected in Taian Central Hospital from June 2013 to May 2018. Eight of the 72 patients were lost during the follow-up stage, and 64 patients were ultimately utilized. The included patients were diagnosed as glioma by postoperative pathology and were graded according to WHO classification and the grading standard of nervous system tumors (2016). None of the patients received any form of radiotherapy, chemotherapy, targeted therapy, immunotherapy, or any other anti-cancer treatments prior to operation, and each study participant signed the informed consent form before sampling. The ethics committee of the Taian Central Hospital approved this study. All methods were performed in accordance with the relevant guidelines and regulations.

\section{Cell Culture.}

U251 was purchased from Shanghai Cell Bank at the Chinese Academy of Medical Sciences (Shanghai, China), and A172 from the Central Laboratory of Taian Central Hospital. Both cell lines were cultured in a DMEM medium (GIBCO, Gaithersburg, MD, USA) which contained $10 \%$ fetal bovine serum (FBS, GIBCO) and $0.2 \%$ penicillin streptomycin (Invitrogen, Carlsbad, California, USA). It was cultured in an incubator at $37^{\circ} \mathrm{C}$, with $95 \%$ humidity and $5 \% \mathrm{CO} 2$.

\section{Real Time Quantitative PCR (qRT-PCR).}

Following the instructions of the miRNeasy Mini Kit (Qiagen, Duesseldorf, Nordrhein-Westfalen, Germany), total RNA was extracted from glioma tissue, normal brain tissue, and glioma cells. Total RNA was extracted from plasma by the miRNeasy Serum/Plasma Kit (Qiagen), and FFPE tissue samples were extracted using the miRNeasy FFPE Kit (Qiagen). The reverse transcription experiment was carried out by using miscript II RT Kit (Qiagen) to synthesize cDNA. The miscript SYBR Green PCR Kit (Qiagen) was implemented to measure the qRT-PCR reaction of miRNA. Primer sequences were used as follows: miR- 
30c Forward: 5'-TGTGTTTTTATTGTTTTTGTTGTCCCA-3'. miR-30c Reverse: 5'GGGACAGAACAGGTTAATGGGAA-3'. U6-forward: 5'-GCTTCGGCAGCACATATACT- AAAAT-3'. U6-reverse: 5'-CGCTTCACGAATTTGCGTGTCAT-3'

The reaction was performed in the LightCycler480 qRT-PCR system (Roche molecular systems, Indianapolis, USA), operating according to the instructions. After amplification, the cycle threshold (CT) of each PCR reaction was calculated by measuring the number of cycles required when the CDNA template reaches the set fluorescence intensity by PCR reaction. Three multiple wells were set in each sample, and U6 was used as an internal reference to normalize the target gene. The relative expression of miR-1 was determined according to $2^{-\Delta \Delta C t}$. TB Green ${ }^{\text {TM }}$ Premix Ex Taq ${ }^{\text {TM }}$ II (Tli RNaseH plus) (Takara, Japan) was used to analyze the PCR reaction of mRNA. $\beta$-actin was used as an internal reference. The primer sequences used were as follows:

Notch1 Forward: 5'-GCTACAACTGCGT GTGTGTC-3'. Notch1 Reverse: 5'-GTTGGTGTC- GCAGTTGGAGC-3'. $\beta$-actin Forward: 5'-CAAAGGCCAACAGAGAGAAGAT-3'. $\beta$-actin Reverse: 5'-TGAGACACACCATCACCAGAAT$3^{\prime}$. The relative expression of Notch1 was determined according to $2^{-\Delta \Delta C t}$.

\section{Cell Transfection.}

In the six well plates, as glioma cells reached $70-80 \%$ confluence, transfection was executed. Mimics that increased expression levels of miR-30c or negative control (NC) inhibitor suppresed the expression levels of miR-30c or inhibitor negative control (inhibitor NC), and shRNA inhibiting Notch1 expression or shRNA negative control (shRNA NC) (Gemma, Shanghai, China) were diluted by Opti-MEM ${ }^{\text {TM }}$ to a suitable concentration. Next, Lipofectamine ${ }^{\mathrm{TM}} 3000$ transfection reagent (Invitrogen) was added. After being incubated for 10-15 minutes at room temperature, the mixture was added to the well plates. After transfection for $24-72$, the cells were gathered and reserved for later experiments.

\section{Cell Proliferation, Migration, and Invasion Experiments.}

he cell proliferation of miR-30c was detected by the CCK-8 (Cell Counting Kit-8, Beyotime, Shanghai, China) assay. The logarithmic growth phase glioma cells were transferred into 96 well plates, with about $2 \times 10^{3}$ cells per well. At $0,24,48$, and 72 hours post-transfection, $10 \mu \mathrm{l}$ CCK- 8 cells were added into each well. After adding CCK-8, the cells were allocated in an incubator at $37^{\circ} \mathrm{C}$, with $5 \% \mathrm{CO} 2$. After 2 hours, the absorbency was detected at $450 \mathrm{~nm}$ by a microplate reader.

The cell migration experiment was carried out in a Transwell chamber with 24-pore plates and $8 \mu \mathrm{m}$ holes. DMEM medium containing $20 \%$ fetal bovine serum was added to the plates. Next, $1 \times 10^{5}$ transfected glioma cells (200ul, serum-free medium) were added to the upper chamber. After 24 hours, the glioma cells reached the opposite side of the membrane through the pores on the filter membrane, and were then fixed with methanol for 15 minutes and stained with $0.1 \%$ crystal violet for 20 minutes. Five visual fields 
were selected to count at random. Matrigel (Matrigel/ DMEM= 8:1, BD, Franklin, NJ, USA) was added to the upper chamber, with each chamber containing 60ul. The same method was employed for the cell invasion experiment, and fixation and staining were performed at 48 hours.

\section{Luciferase Reporter Assay.}

A172 and U251 glioma cells were placed into 96 well plates. The plasmid of Notch1 wild type vector (pGL3-Notch1-3'-UTR-WT) or mutant vector (pGL3-Notch1-3'-UTR-Mut) and miR-30c mimics or NC were cotransfected with Lipofectamine ${ }^{\mathrm{TM}} 3000$ (Invitrogen). At 48 hours after transfection, the luciferase activity was measured using the dual luciferase reporter system (Promega, Madison, WI). The ratio of firefly to Renilla luciferase activity was defined as normalized luciferase activity.

\section{Western Blotting.}

Cell lysate was used to treat cells and tissues. Protease inhibitor PMSF (Beyotime) was added. The BCA method was used to measure protein concentration. Proteins were extracted by SDS-PAGE, and then transferred to nitrocellulose membranes. The nitrocellulose membranes were blocked with $5 \%$ skimmed milk prepared with TBST and subsequently incubated with the diluted primary antibodies, anti-Notch1, 1:1000, anti-Vimentin, 1:1000, anti-E-cadherin, 1:1000, anti-E-cadherin, 1:1000 (cell signaling technology, CST, Boston, USA), at $4^{\circ} \mathrm{C}$ incubation overnight. After TBST was eluted on three occasions, horseradish peroxidase labeled anti-rabbit or anti-mouse secondary antibodies (CST) was added and shaken on a shaking table for 1 hour at room temperature. Then, the target bands were imaged using the chemiluminescence detection system (EMD, millipore, Billerica, Ma, USA) and analyzed quantitatively by Image $\mathrm{J}$ software (National Institutes of health, Bethesda, MD, USA). $\beta$-Actin (1:5000) was implemented as internal reference.

\section{Statistical Analysis.}

The experimental results were expressed as means \pm SEM. An Independent-Samples T test was implemented to detect the differences among glioma tissues and normal tissues after brain trauma decompression, preoperative plasma of glioma patients, and plasma of healthy volunteers. ANOVA was used to evaluate the expression of miR-30c in varied clinicopathological features. Next, Pearson analysis was used to evaluate the connection between miR-30c expression and the clinicopathological features. Cytological experiments were then analyzed by a Paired-Samples $T$ test. A result of $P<0.05$ was considered statistically significant, and $\mathrm{P}<0.01$ was considered remarkably statistically significant.

\section{Declarations}

\section{Acknowledgements}


This study was supported by the Shandong Medicine and Health Science Technology Expansion Plan, China [No. 2017WS069] and the Taian Science Technology Expansion Plan, Shandong, China [Nos. 2018NS0117 and 2017NS0147].

\section{Author Contributions}

J.X. designed the study, supervised the research and reviewed the manuscript. M.K.L. and W.Z.L. finished the experiments and wrote the manuscript. M.K.L. performed the statistical analysis of the data. All authors reviewed the manuscript.

\section{Competing interests}

The authors declare no competing interests.

\section{References}

1. Brandao, M., Simon, T., Critchley, G. \& Giamas, G. Astrocytes, the rising stars of the glioblastoma microenvironment. Glia, 67, 779-790 https://doi.org/10.1002/glia.23520 (2019).

2. Lu, W. \& Kang, Y. Epithelial-Mesenchymal Plasticity in Cancer Progression and Metastasis. Dev Cell, 49, 361-374 https://doi.org/10.1016/j.devcel.2019.04.010 (2019).

3. Sommariva, M. \& Gagliano, N. E-Cadherin in Pancreatic Ductal Adenocarcinoma: A Multifaceted Actor during EMT., 9, https://doi.org/10.3390/cells9041040 (2020).

4. Wong, S. H. M., Fang, C. M., Chuah, L. H., Leong, C. O. \& Ngai, S. C. E-cadherin: Its dysregulation in carcinogenesis and clinical implications. Crit Rev Oncol Hematol, 121, 11-22 https://doi.org/10.1016/j.critrevonc.2017.11.010 (2018).

5. Tian, H. et al. AKT-induced IncRNA VAL promotes EMT-independent metastasis through diminishing Trim16-dependent Vimentin degradation. Nat Commun, 11, 5127 https://doi.org/10.1038/s41467020-18929-0 (2020).

6. Loh, C. Y. et al. The E-Cadherin and N-Cadherin Switch in Epithelial-to-Mesenchymal Transition: Signaling, Therapeutic Implications, and Challenges., 8, https://doi.org/10.3390/cells8101118 (2019).

7. Arefin, B., Parvin, F., Bahrampour, S., Stadler, C. B. \& Thor, S. Drosophila Neuroblast Selection Is Gated by Notch, Snail, SoxB, and EMT Gene Interplay. Cell Rep, 29 (e3633), 3636-3651 https://doi.org/10.1016/j.celrep.2019.11.038 (2019).

8. Yuan, X. et al. Notch signaling and EMT in non-small cell lung cancer: biological significance and therapeutic application. J Hematol Oncol, 7, 87 https://doi.org/10.1186/s13045-014-0087-z (2014).

9. Hibdon, E. S. et al. Notch and mTOR Signaling Pathways Promote Human Gastric Cancer Cell Proliferation. Neoplasia, 21, 702-712 https://doi.org/10.1016/j.neo.2019.05.002 (2019). 
10. Bigas, A. \& Espinosa, L. The multiple usages of Notch signaling in development, cell differentiation and cancer. Curr Opin Cell Biol, 55, 1-7 https://doi.org/10.1016/j.ceb.2018.06.010 (2018).

11. Chen, L., Wang, J., Li, J. W., Zhao, X. W. \& Tian, L. F. LncRNA MEG3 inhibits proliferation and promotes apoptosis of osteosarcoma cells through regulating Notch signaling pathway. Eur Rev Med Pharmacol Sci, 24, 581-590 https://doi.org/10.26355/eurrev_202001_20034 (2020).

12. Baisiwala, S. et al. LNX1 Modulates Notch1 Signaling to Promote Expansion of the Glioma Stem Cell Population during Temozolomide Therapy in Glioblastoma. Cancers (Basel), 12, https://doi.org/10.3390/cancers12123505 (2020).

13. Li, J. et al. Notch1 is an independent prognostic factor for patients with glioma. J Surg Oncol, 103, 813-817 https://doi.org/10.1002/jso.21851 (2011).

14. Natsuizaka, M. et al. Interplay between Notch1 and Notch3 promotes EMT and tumor initiation in squamous cell carcinoma. Nat Commun, 8, 1758 https://doi.org/10.1038/s41467-017-01500-9 (2017).

15. Li, J. et al. Targeting the Notch1 oncogene by miR-139-5p inhibits glioma metastasis and epithelialmesenchymal transition (EMT). BMC Neurol, 18, 133 https://doi.org/10.1186/s12883-018-1139-8 (2018).

16. Zeng et al. Inhibition of Notch1 reverses EMT and chemoresistance to cisplatin via direct downregulation of MCAM in triple-negative breast cancer cells. Int J Cancer, 147, 490-504 https://doi.org/10.1002/ijc.32911 (2020).

17. Liu, J. et al. Mir-758-5p Suppresses Glioblastoma Proliferation, Migration and Invasion by Targeting ZBTB20. Cell Physiol Biochem, 48, 2074-2083 https://doi.org/10.1159/000492545 (2018).

18. Villanova, L. et al. miR-1285-3p Controls Colorectal Cancer Proliferation and Escape from Apoptosis through DAPK2. Int J Mol Sci, 21, https://doi.org/10.3390/ijms21072423 (2020).

19. Liu, W. et al. MicroRNA-1 suppresses proliferation, migration and invasion by targeting Notch2 in esophageal squamous cell carcinoma. Sci Rep, 8, 5183 https://doi.org/10.1038/s41598-018-23421-3 (2018).

20. Lu, G. D., Cheng, P., Liu, T. \& Wang, Z. BMSC-Derived Exosomal miR-29a Promotes Angiogenesis and Osteogenesis. Front Cell Dev Biol, 8, 608521 https://doi.org/10.3389/fcell.2020.608521 (2020).

21. Zhu, C., Huang, Q. \& Zhu, H. miR-383 Inhibited the Cell Cycle Progression of Gastric Cancer Cells via Targeting Cyclin E2. DNA Cell Biol, 38, 849-856 https://doi.org/10.1089/dna.2019.4624 (2019).

22. Song, $\mathrm{S}$. et al. Urinary exosome miR-30c-5p as a biomarker of clear cell renal cell carcinoma that inhibits progression by targeting HSPA5. J Cell Mol Med, 23, 6755-6765 https://doi.org/10.1111/jcmm.14553 (2019).

23. Pei, B. et al. Downregulation of microRNA-30c-5p was responsible for cell migration and tumor metastasis via COTL1-mediated microfilament arrangement in breast cancer. Gland Surg, 9, 747758 https://doi.org/10.21037/gs-20-472 (2020).

24. Baraniskin, A. et al. MicroRNA-30c as a novel diagnostic biomarker for primary and secondary B-cell lymphoma of the CNS. J Neurooncol, 137, 463-468 https://doi.org/10.1007/s11060-018-2749-0 
(2018).

25. Jafarzadeh, M., Mohammad Soltani, B., Ekhteraei Tousi, S. \& Behmanesh, M. Hsa-miR-497 as a new regulator in TGFbeta signaling pathway and cardiac differentiation process., $675,150-156$ https://doi.org/10.1016/j.gene.2018.06.098 (2018).

26. Chen, P., Chen, F., Lei, J., Li, Q. \& Zhou, B. Activation of the miR-34a-Mediated SIRT1/mTOR Signaling Pathway by Urolithin A Attenuates D-Galactose-Induced Brain Aging in Mice., 16, 1269-1282 https://doi.org/10.1007/s13311-019-00753-0 (2019).

27. Qian, Z. et al. Excess administration of miR-340-5p ameliorates spinal cord injury-induced neuroinflammation and apoptosis by modulating the P38-MAPK signaling pathway. Brain Behav Immun, 87, 531-542 https://doi.org/10.1016/j.bbi.2020.01.025 (2020).

28. Onyshchenko, K. V. et al. Expression of micro-RNA hsa-miR-30c-5p and hsa-miR-138-1 in renal cell carcinoma. Exp Oncol, 42, 115-119 https://doi.org/10.32471/exp-oncology.2312-8852.vol-42-no$2.14632(2020)$.

29. Herichova, I., Reis, R., Hasakova, K. \& Vician, M. Downregulation of miR-30c-5p expression in colorectal cancer tissue is sex-dependent. Physiol Res, 69, S479-S487 https://doi.org/10.33549/physiolres.934598 (2020).

30. Cao, J. M., Li, G. Z., Han, M., Xu, H. L. \& Huang, K. M. MiR-30c-5p suppresses migration, invasion and epithelial to mesenchymal transition of gastric cancer via targeting MTA1. Biomed Pharmacother, 93, 554-560 https://doi.org/10.1016/j.biopha.2017.06.084 (2017).

31. Yuan, R. X., Bao, D. \& Zhang, Y. Linc00707 promotes cell proliferation, invasion, and migration via the miR-30c/CTHRC1 regulatory loop in breast cancer. Eur Rev Med Pharmacol Sci, 24, 4863-4872 https://doi.org/10.26355/eurrev_202005_21175 (2020).

32. Liu, S., Li, X. \& Zhuang, S. miR-30c Impedes Glioblastoma Cell Proliferation and Migration by Targeting SOX9. Oncol Res, 27, 165-171 https://doi.org/10.3727/096504018X15193506006164 (2019).

33. Sun, Z. et al. Glioblastoma Stem Cell-Derived Exosomes Enhance Stemness and Tumorigenicity of Glioma Cells by Transferring Notch1 Protein. Cell Mol Neurobiol, 40, 767-784 https://doi.org/10.1007/s10571-019-00771-8 (2020).

34. Man, J. et al. Hypoxic Induction of Vasorin Regulates Notch1 Turnover to Maintain Glioma Stem-like Cells. Cell Stem Cell 22, 104-118 e106, doi:10.1016/j.stem.2017.10.005 (2018).

35. Gu, G. et al. NKAP alters tumor immune microenvironment and promotes glioma growth via Notch1 signaling. J Exp Clin Cancer Res, 38, 291 https://doi.org/10.1186/s13046-019-1281-1 (2019).

36. Vinchure, O. S. et al. Polycomb complex mediated epigenetic reprogramming alters TGF-beta signaling via a novel EZH2/miR-490/TGIF2 axis thereby inducing migration and EMT potential in glioblastomas. Int J Cancer, 145, 1254-1269 https://doi.org/10.1002/ijc.32360 (2019).

37. Zhao, C. et al. MicroRNA-128-3p Enhances the Chemosensitivity of Temozolomide in Glioblastoma by Targeting c-Met and EMT. Sci Rep, 10, 9471 https://doi.org/10.1038/s41598-020-65331-3 (2020). 
A

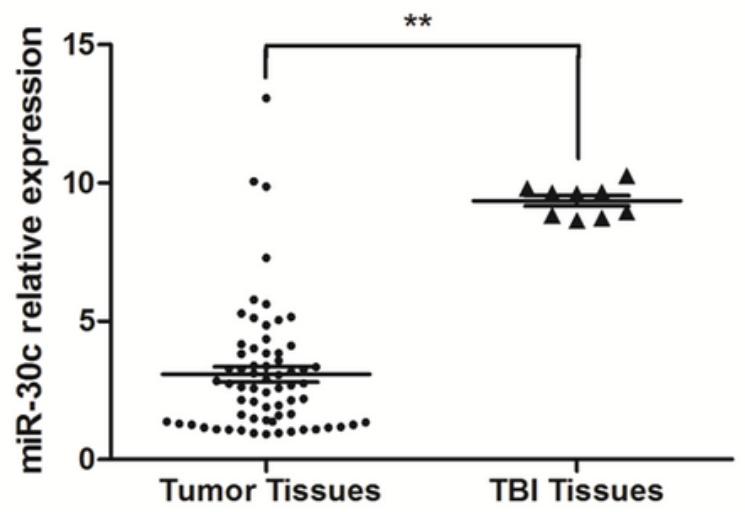

C
B

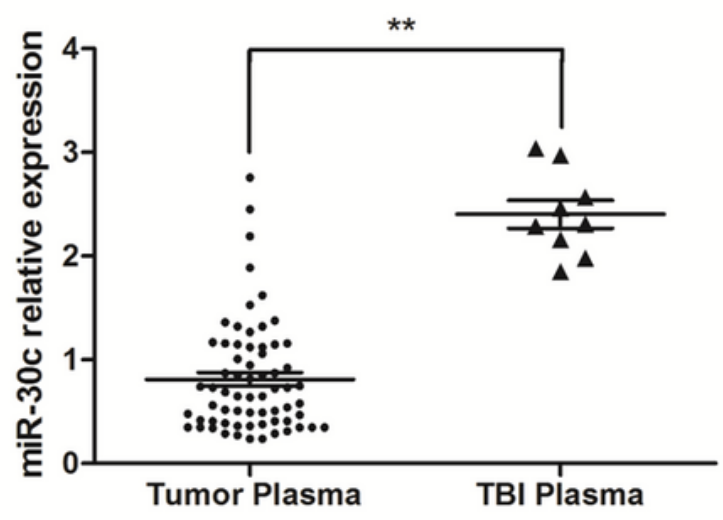

miR-30c relative expression

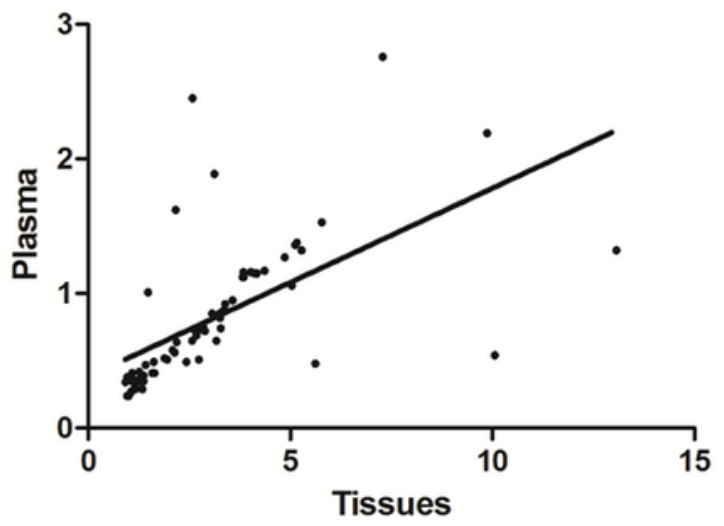

D

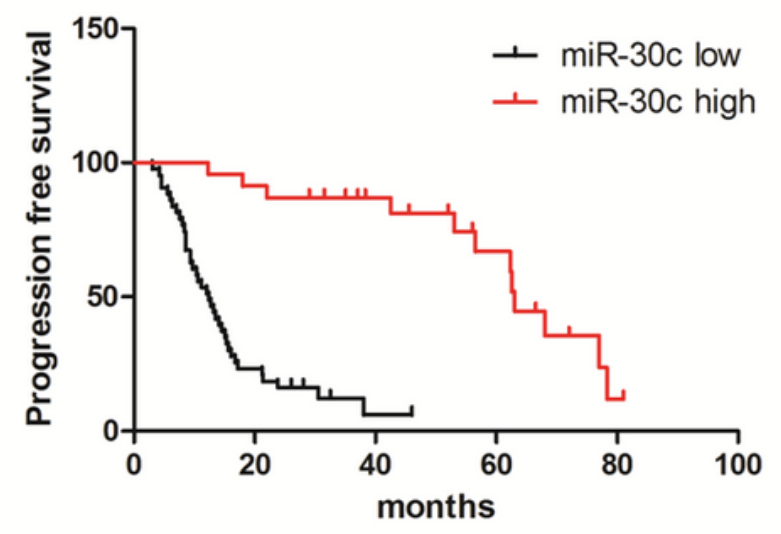

E

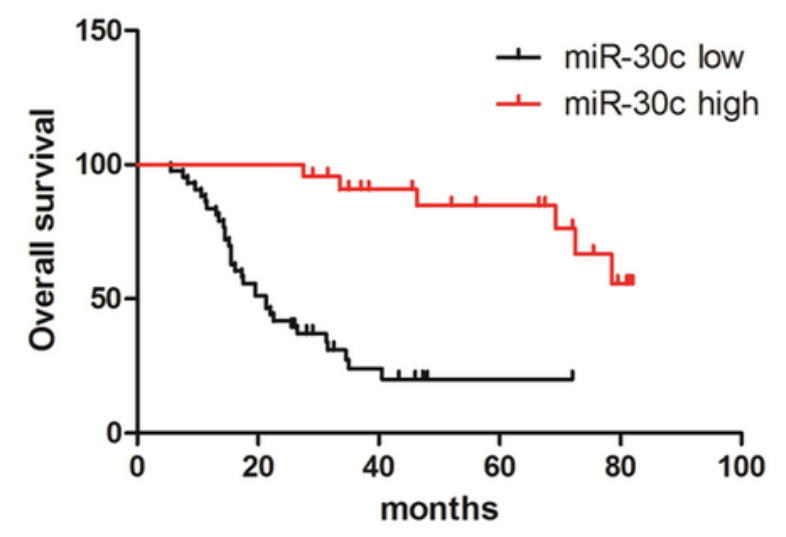

Figure 1

The expression levels of miR-30c in human glioma tissue, preoperative plasma, and paraffin specimens. (A) The expression levels of miR-30c in glioma tissues were found to be significantly lower than the levels found in normal brain injury tissue $(* * \mathrm{P}<0.01)$. (B) The expression levels of miR-30c in plasma of 
patients with glioma prior to operation was significantly lower than the levels found in healthy volunteers (**P $<0.01$ ). (C) The correlation analysis of miR-30c in glioma tissue and plasma. (D, E) PFS and OS of patients with a high expression of miR-30c were significantly longer than those of patients with a low expression of miR-30c $(P<0.01)$.

A

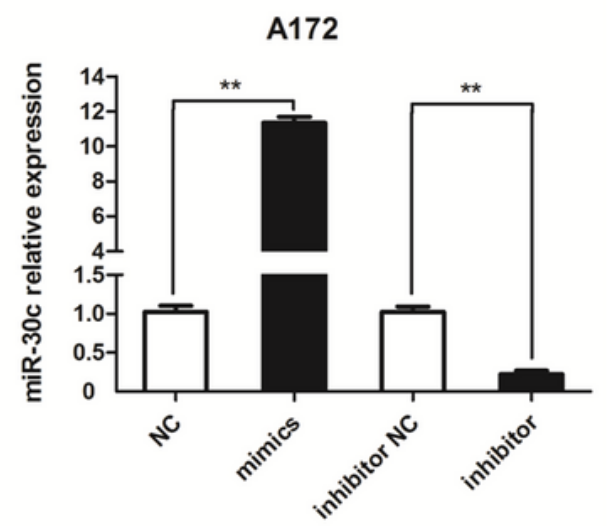

$\mathrm{B}$

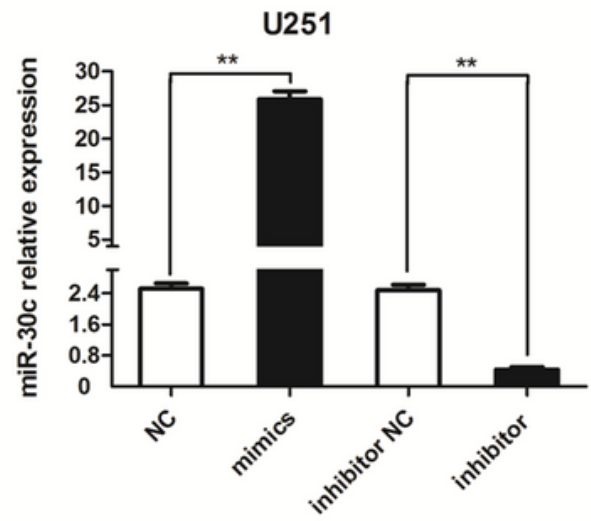

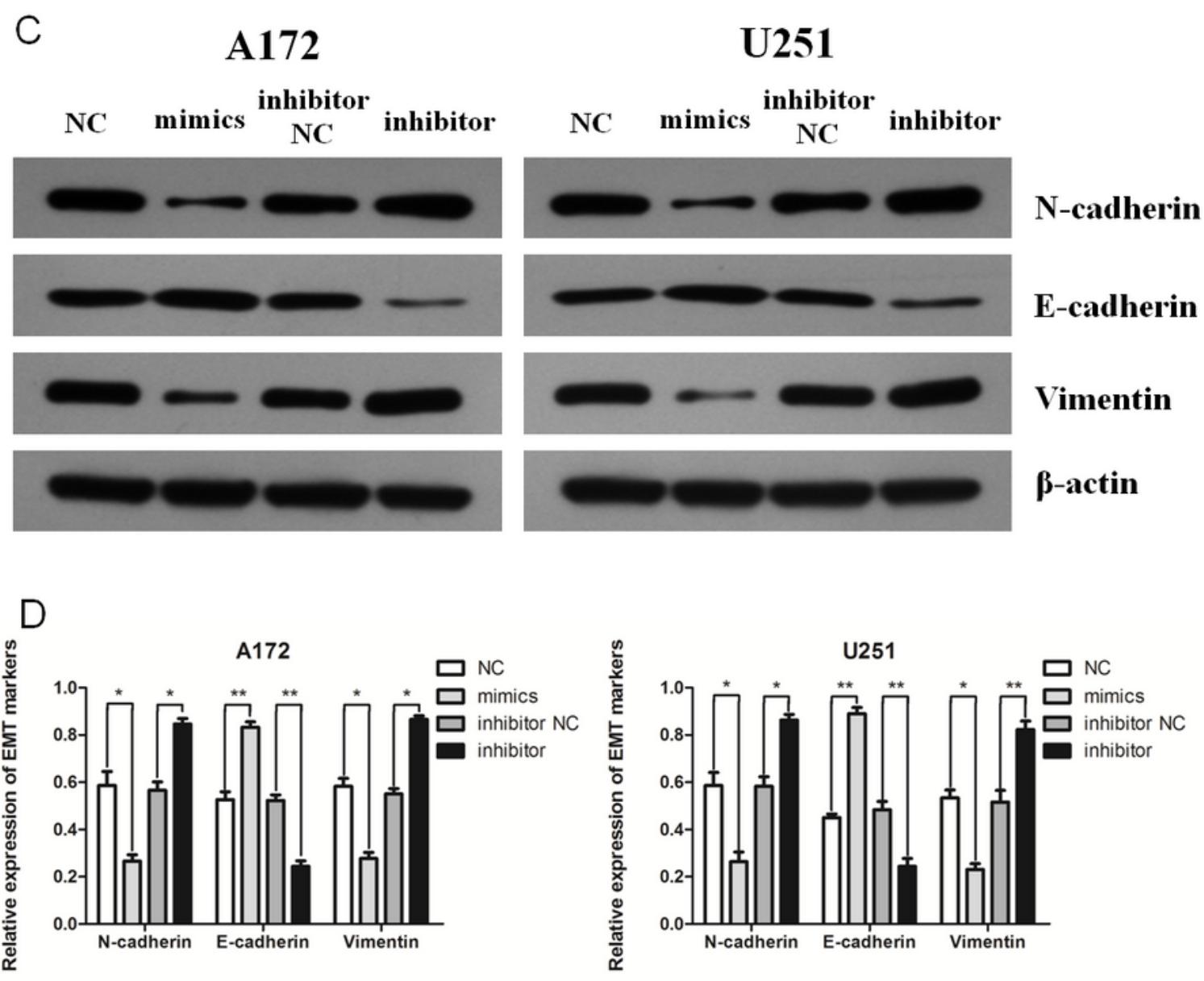

Figure 2 
miR-30c inhibits EMT process of glioma cells. (A, B) QRT-PCR was used to verify the change of miR-30c expression level of A172 and U251 after transfection of the mimics and the inhibitor (**P<0.01). (C, D) the EMT process of glioma cells was changed after the transfection of mimics and the inhibitor $\left({ }^{*} \mathrm{P}<\right.$ $0.05, * * \mathrm{P}<0.01)$.

A

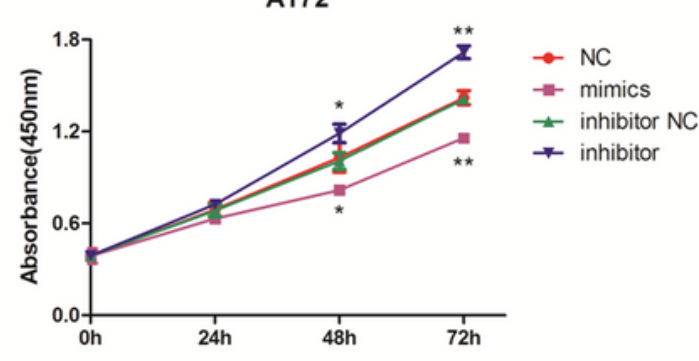

C

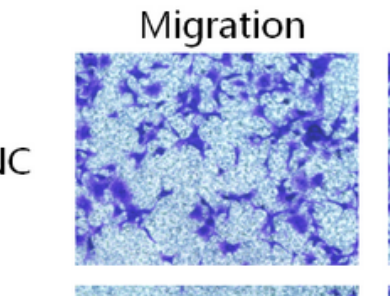

mimics

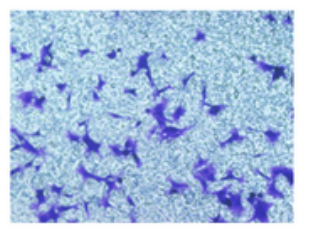

inhibitor

NC

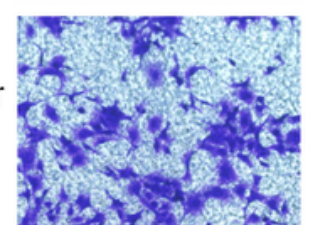

inhibitor

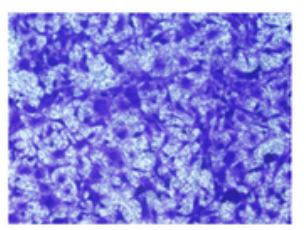

E

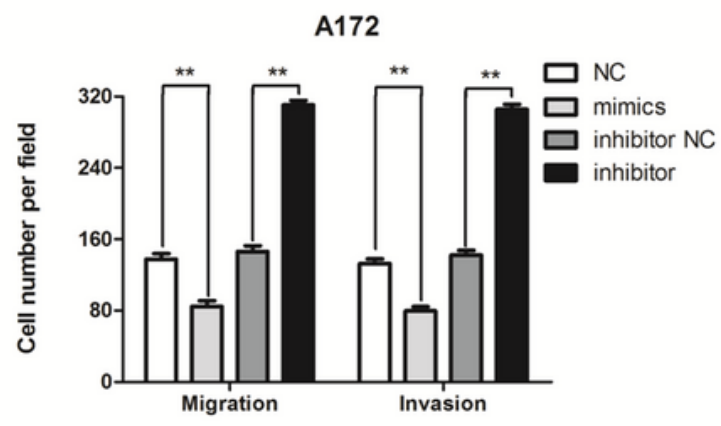

B

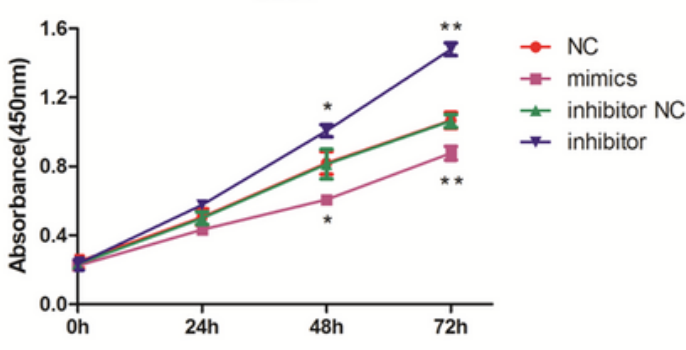

U251
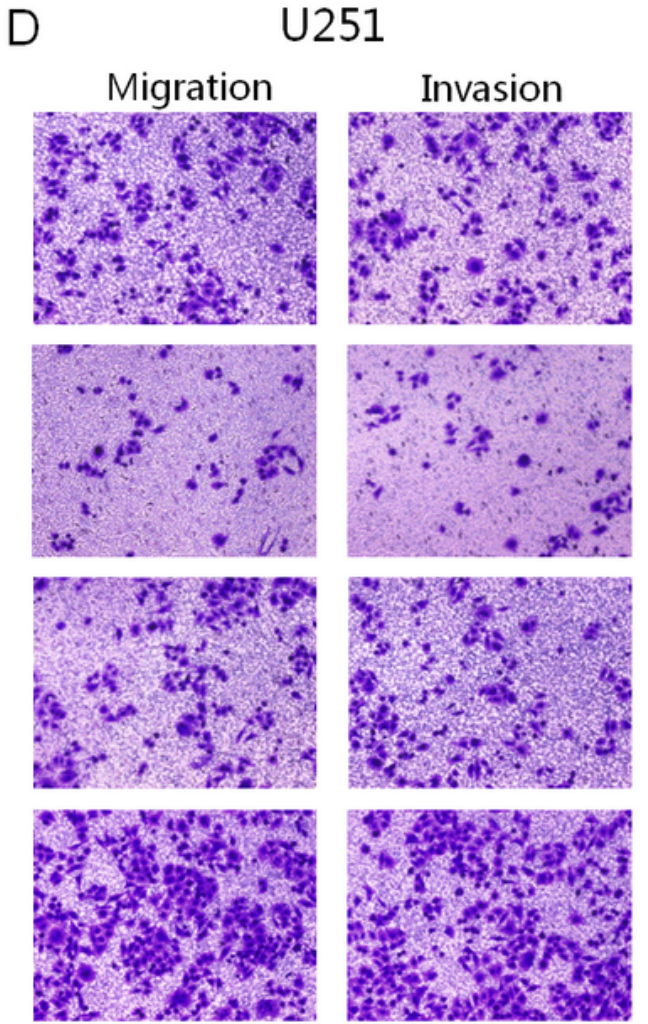

F

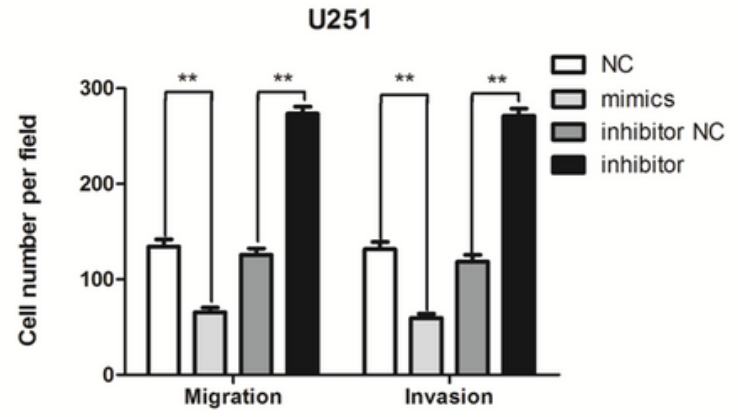

Figure 3 
miR-30c reduces the proliferation, migration, and invasion of glioma cells. (A, B) CCK8 assay revealed that miR-30c could impact the proliferation ability of $A 172$ and $U 251$ cells ( ${ }^{*}<<0.05$, $\left.{ }^{*} P<0.01\right)$. (C, E) The migration and invasion abilities of mimics and inhibitor groups were significantly changed in A172 $(* * P<0.01)$. (D, F) The migration and invasion abilities of U251 were significantly changed after the transfection of mimics and inhibitor $(* \star P<0.01)$.

A

Position 1520-1526 of NOTCH1 3' UTR

has-miR-30c-5p

B
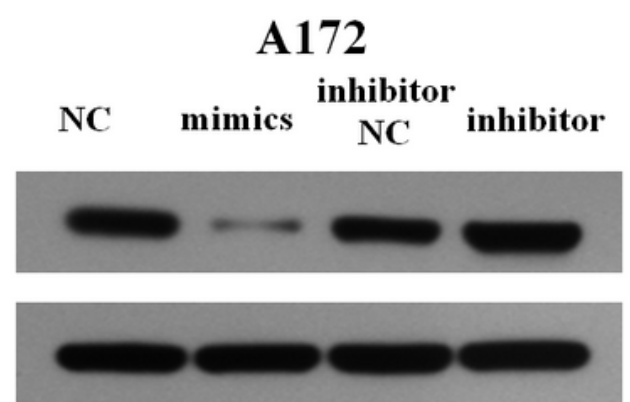

C

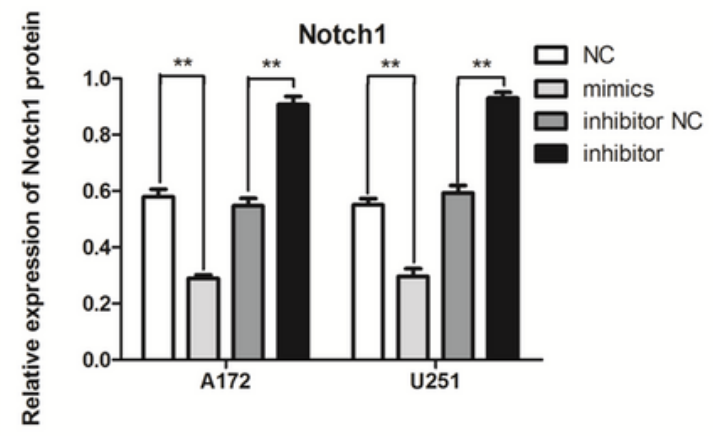

$E$

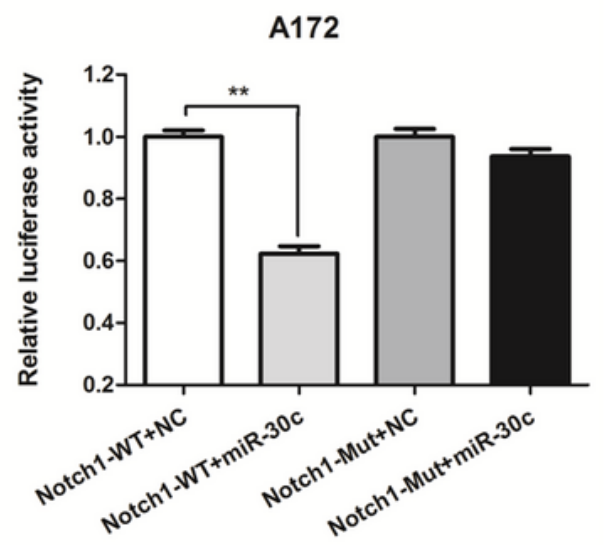

5' AUAAAAUAGAgUgUAGUUUACAG

|| |||||

3' CGACUCUCACAUCCUACAAAUGU

U251

NC mimics $\begin{gathered}\text { inhibitor } \\ \text { NC }\end{gathered}$ inhibitor

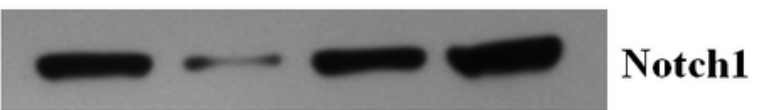

$\boldsymbol{\beta}$-actin

D

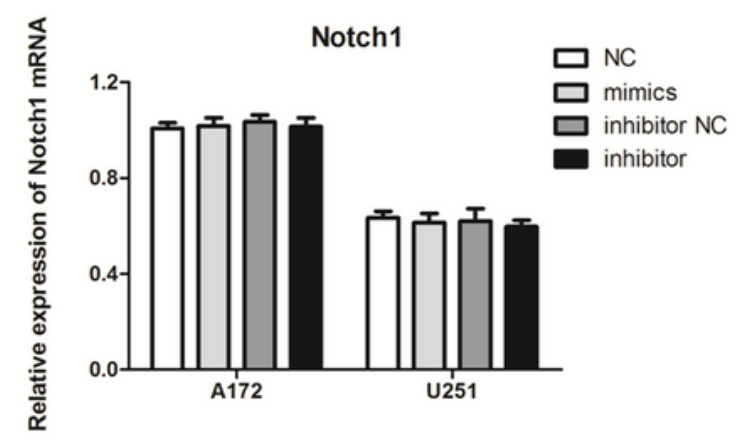

F

U251

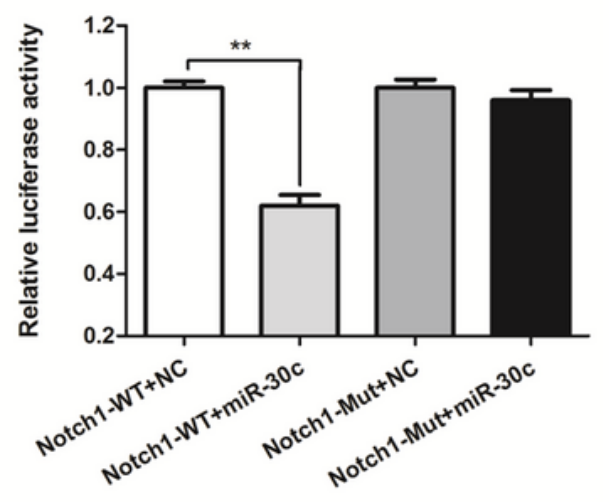

Figure 4 
Notch1 is the direct target gene of miR-30c in glioma. (A) The 3'-UTR region of Notch1 binds to miR-30c. $(B, C)$ The change of the Notch1 protein after transfection of mimics and inhibitor (**P<0.01). (D) There is no alteration in Notch1 mRNA after the transfection of mimics and inhibitor. $(E, F)$ Dual luciferase assay of two kinds of glioma cells $(* * \mathrm{P}<0.01)$.

A

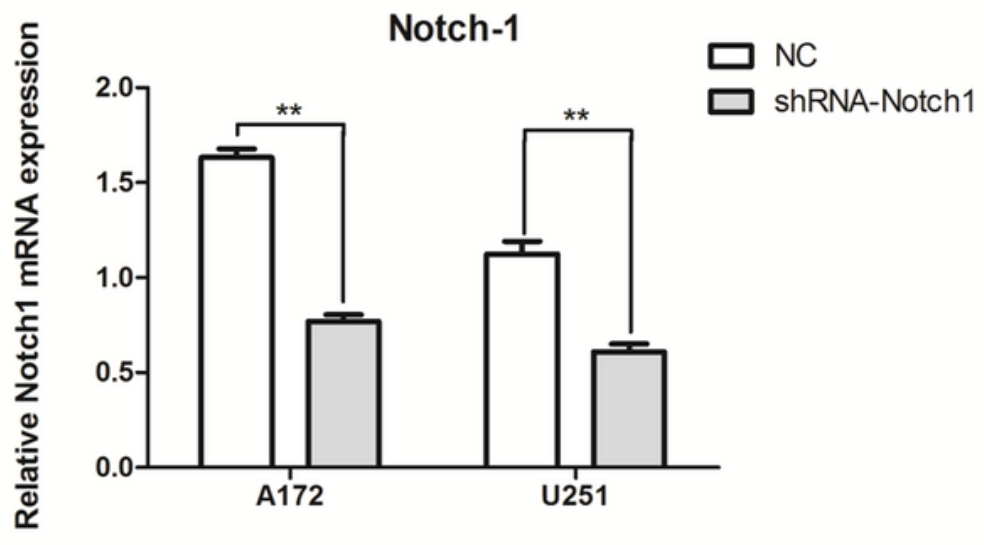

B

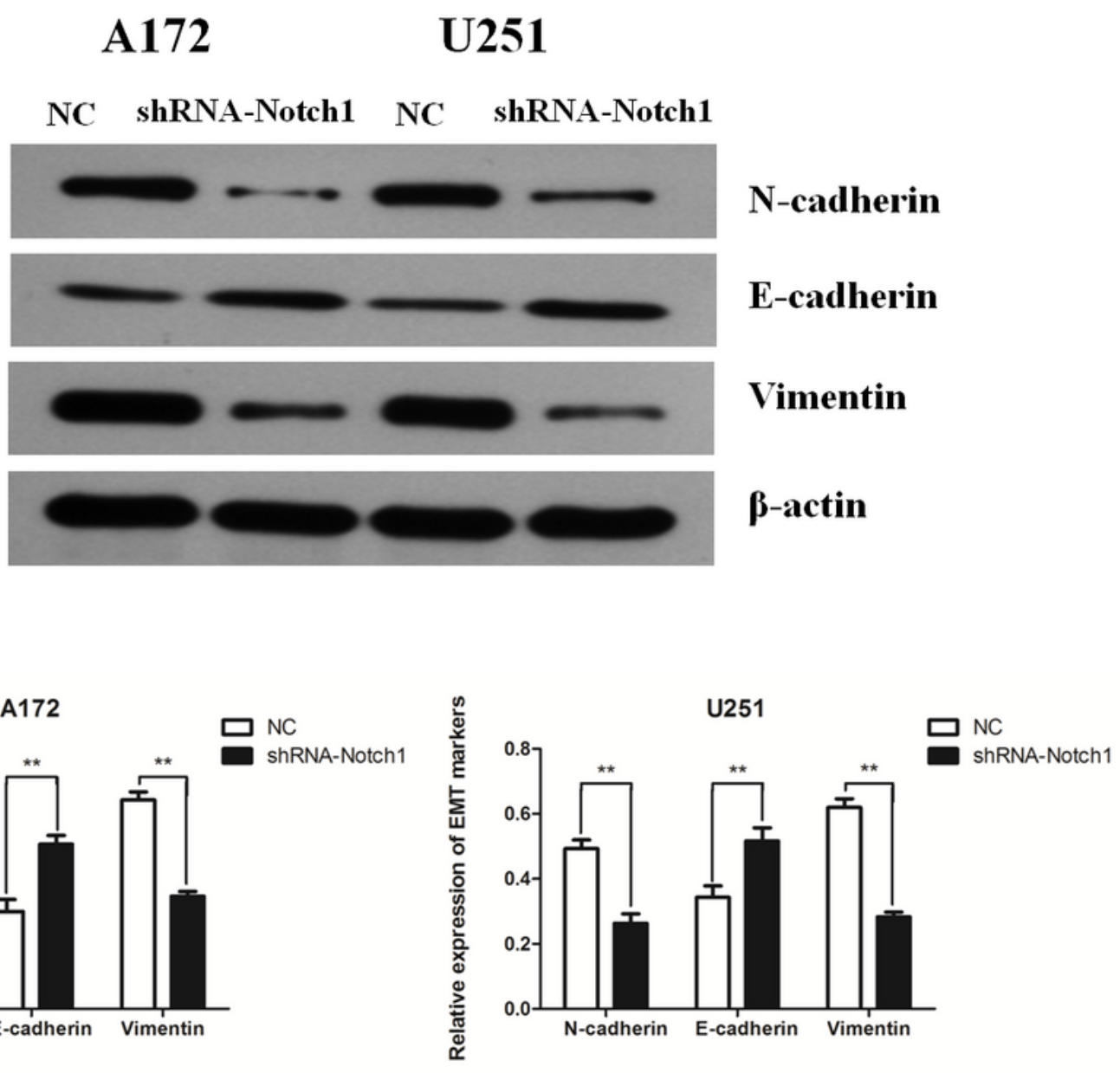

Figure 5 
Notch1 can promote the EMT process of glioma. (A) The changes of Notch1 mRNA after shRNA-Notch1 transfection were verified by qRT-PCR $(* * P<0.01)$. $(B, C)$ The changes of EMT related proteins after shRNA-Notch1 transfection (**P $<0.01)$.
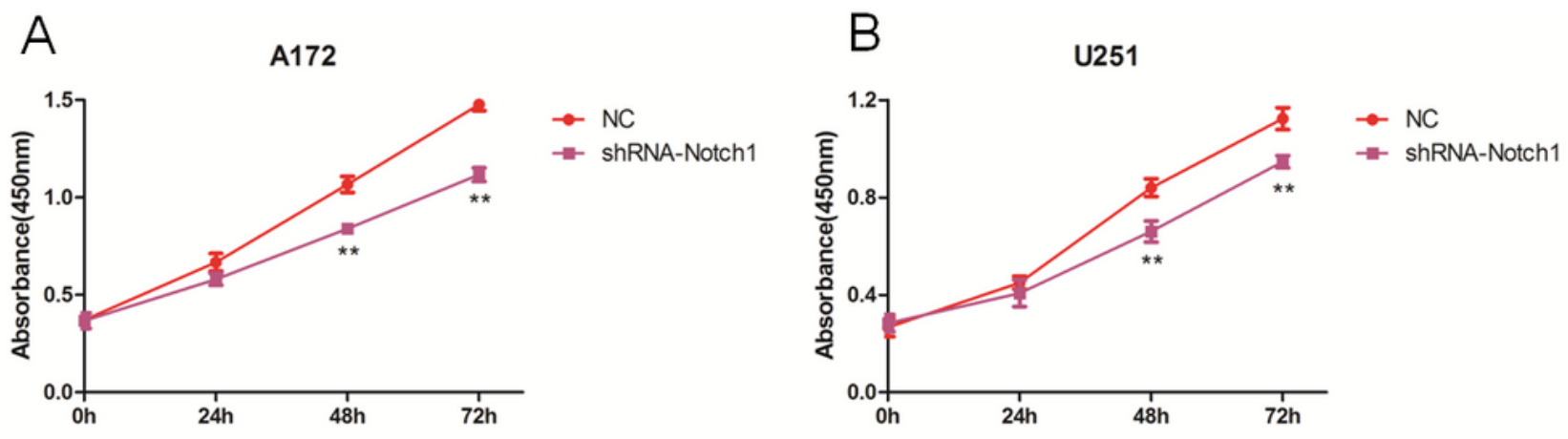

C

A172
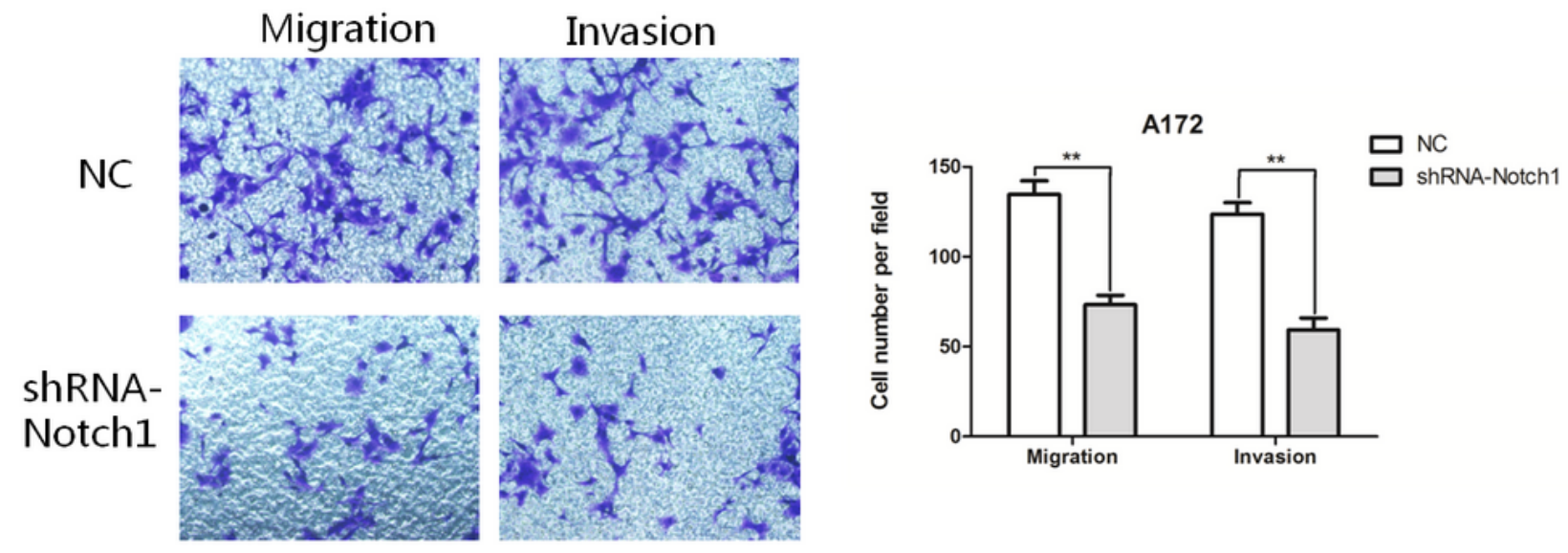

$\mathrm{D}$

\section{U251}
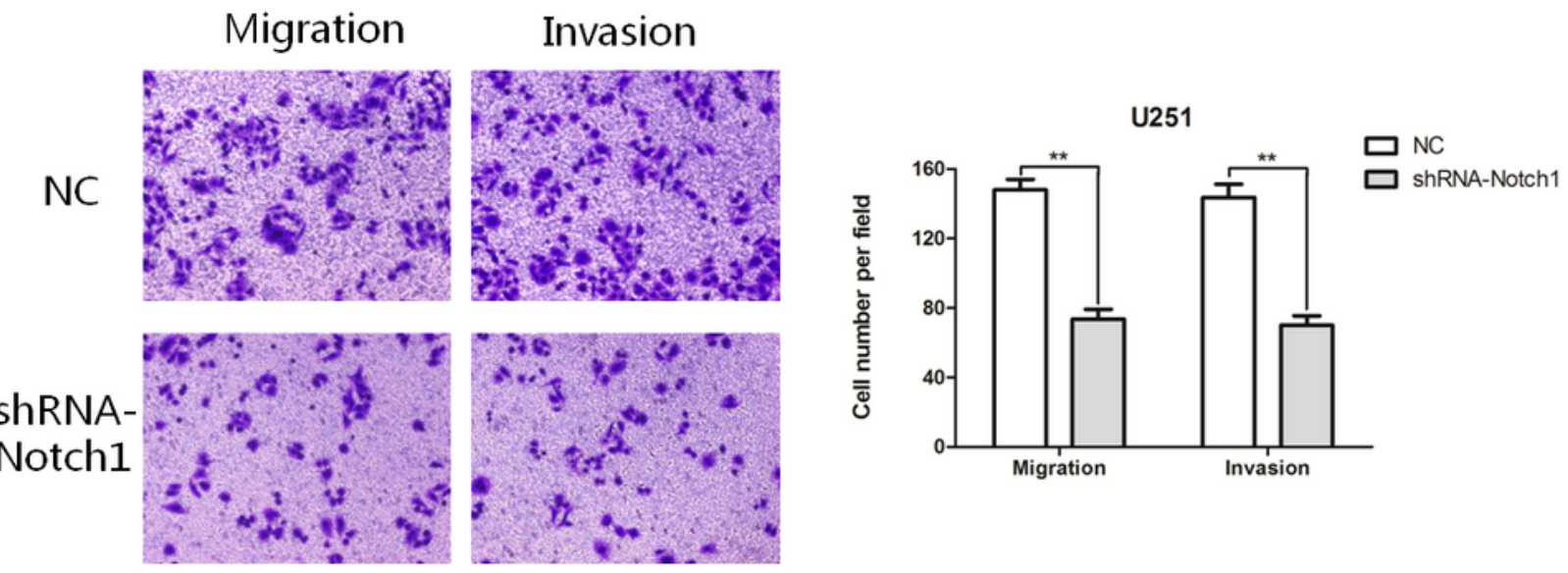

Figure 6

Notch1 can promote the proliferation, invasion, and migration of glioma. (A, B) CCK8 assay showed that Notch1 could impact the proliferation ability of A172 and U251 cells (**P $<0.01)$. (C, D) Notch1 could 
change cell migration and invasion ability $(* * P<0.01)$.

A

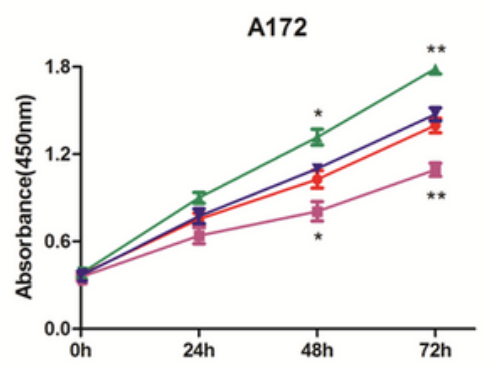

C

\section{A172}

NC+shRNA Notch

inhibitor+Notch1 NC

* inhibitor+shRNA Notch1

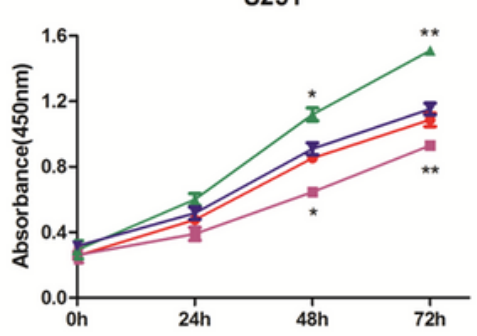

U87

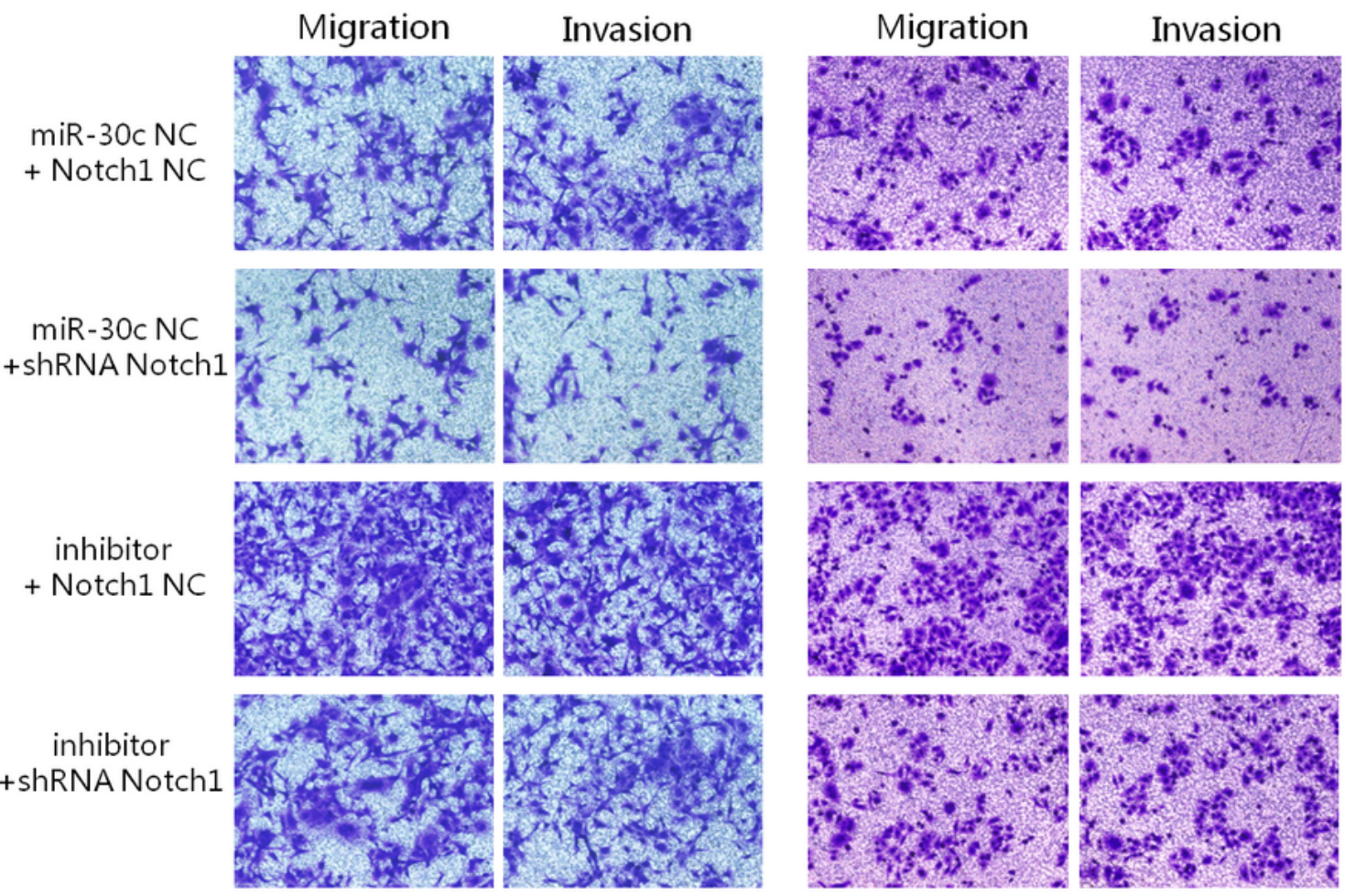

E

$\mathrm{F}$
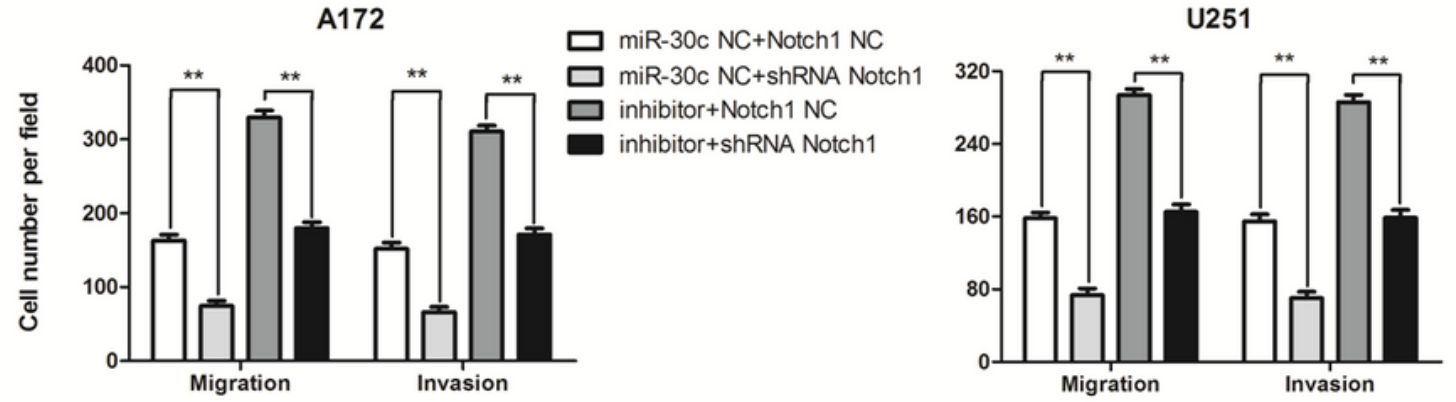

Figure 7

miR-30c hindered the ability of proliferation, migration, and invasion by down-regulating the expression levels of Notch1. (A, B) miR-30c inhibitor facilitated an increase in the proliferation of glioma cells, while shRNA-Notch1 could undo the change $\left({ }^{*} P<0.05,{ }^{*} \mathrm{P}<0.01\right)$. (C, D, E, F) shRNA-Notch1 attenuated the enhancement of migration and invasion of glioma cells by the miR-30c inhibitor $(* * P<0.01)$. 


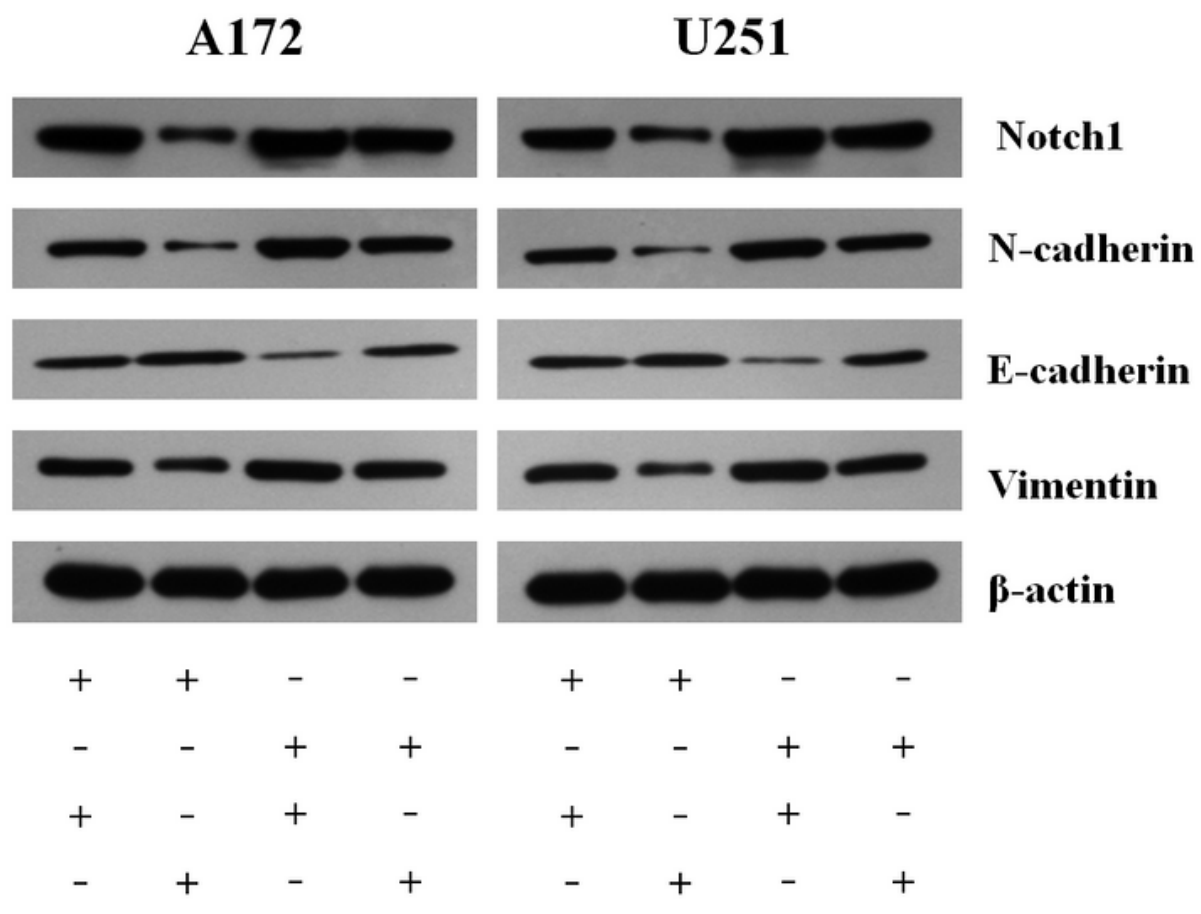

B
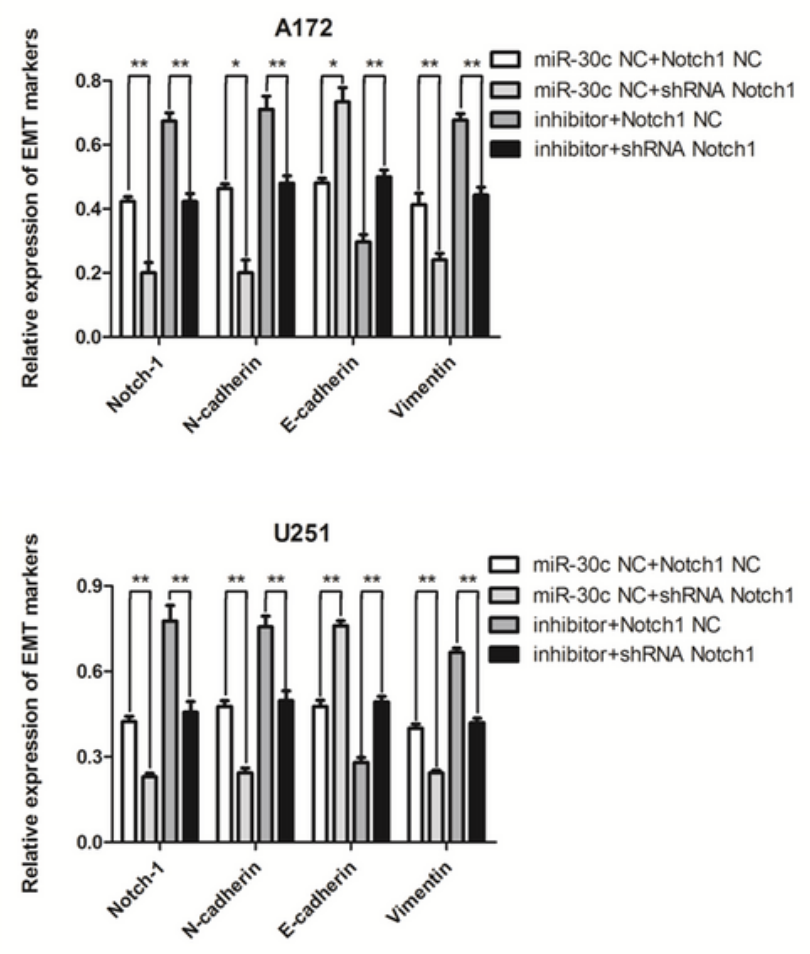

\section{Figure 8}

The miR-30c-mediated EMT depended on Notch1. (A, B) miR-30c inhibitor triggered an increase of Ncadherin and Vimentin and a decrease of E-cadherin, while co-transfection with shRNA-Notch1 partially eliminated these effects $\left({ }^{\star} P<0.05,{ }^{* *} P<0.01\right)$. 